\title{
Optimization of Signal Significance by Bagging Decision Trees *
}

\author{
Ilya Narsky† \\ California Institute of Technology
}

(Dated: September 12, 2018)

\begin{abstract}
An algorithm for optimization of signal significance or any other classification figure of merit suited for analysis of high energy physics (HEP) data is described. This algorithm trains decision trees on many bootstrap replicas of training data with each tree required to optimize the signal significance or any other chosen figure of merit. New data are then classified by a simple majority vote of the built trees. The performance of this algorithm has been studied using a search for the radiative leptonic decay $B \rightarrow \gamma l \nu$ at $B A B A R$ and shown to be superior to that of all other attempted classifiers including such powerful methods as boosted decision trees. In the $B \rightarrow \gamma e \nu$ channel, the described algorithm increases the expected signal significance from $2.4 \sigma$ obtained by an original method designed for the $B \rightarrow \gamma l \nu$ analysis to $3.0 \sigma$.
\end{abstract}

PACS numbers: 02.50.Tt, 02.50.Sk, 02.60.Pn.

\footnotetext{
* Work partially supported by Department of Energy under Grant DE-FG03-92-ER40701.

${ }^{\dagger}$ Electronic address: narsky@hep.caltech.edu
} 


\section{INTRODUCTION}

Separation of signal and background is perhaps the most important problem in analysis of HEP data. Various pattern classification tools have been employed in HEP practice to solve this problem. Fisher discriminant [1] and feedforward backpropagation neural networks 2] are the two most popular methods chosen by HEP analysts at present. Alternative algorithms for classification such as decision trees [3], bump hunting [4], and AdaBoost [5] have been recently explored by the HEP community as well [6, 7, 8]. These classifiers can be characterized by such features as predictive power, interpretability, stability and ease of training, CPU time required for training and classifying new events, and others. It is important to remember that the choice of a classifier for each problem should be driven by specifics of the analysis. For example, if the major goal of pattern classification is to achieve a high quality of signal and background separation, flexible classifiers such as AdaBoost and neural nets should be the prime choice. While neural nets generally perform quite well in low dimensions, they become too slow and unstable in high-dimensional problems losing the competition to AdaBoost. If the analyst, however, is mostly concerned with a clear interpretation of the classifier output, decision trees and bump hunting algorithms are a more appealing option. These classifiers produce rectangular regions, easy to visualize in many dimensions.

One of the problems faced by HEP analysts is the indirect nature of available classifiers. In HEP analysis, one typically wants to optimize a figure of merit expressed as a function of signal and background, $S$ and $B$, expected in the signal region. An example of such figure of merit is signal significance, $S / \sqrt{S+B}$, often used by physicists to express the cleanliness of the signal in the presence of statistical fluctuations of observed signal and background. None of the available popular classifiers optimizes this figure of merit directly. CART [3], a popular commercial implementation of decision trees, splits training data into signal- and background-dominated rectangular regions using the Gini index, $Q=2 p(1-p)$, as the optimization criterion, where $p$ is the correctly classified fraction of events in a tree node. Neural networks typically minimize a quadratic classification error, $\mathcal{E}_{\text {qua }}=\sum_{n=1}^{N}\left(y_{n}-\right.$ $\left.f\left(x_{n}\right)\right)^{2}$, where $y_{n}$ is the true class of an event, -1 for background and 1 for signal, $f\left(x_{n}\right)$ is the continuous value of the class label in the range $[-1,1]$ predicted by the neural network, and the sum is taken over $N$ events in the training data set. Similarly, AdaBoost minimizes an

exponential classification error, $\mathcal{E}_{\exp }=\sum_{n=1}^{N} \exp \left(-y_{n} f\left(x_{n}\right)\right)$. These optimization criteria are not necessarily optimal for maximization of the signal significance. The usual solution is to build a neural net or an AdaBoost-based classifier and then find an optimal cut on the continuous output of the classifier to maximize the signal significance. For decision trees, the solution is to construct a decision tree with many terminal nodes and then combine these nodes to maximize the signal significance.

This problem has been partially addressed in my $\mathrm{C}++$ software package for pattern classification 8]. Default implementations of the decision tree and the bump hunting algorithm include both standard figures of merit used for commercial decision trees such as the Gini index and HEP-specific figures of merit such as the signal significance or the signal purity, $S /(S+B)$. The analyst can optimize an arbitrary figure of merit by providing an implementation to the corresponding abstract interface set up in the package.

AdaBoost and the neural net, however, cannot be modified that easily. The functional forms of the classification error are intimately tied to implementations of these two classification algorithms. Finding a powerful method for optimization of HEP-specific figures of 
merit is therefore an open question.

This note describes an algorithm that can be used for direct optimization of an arbitrary figure of merit. Optimization of the signal significance by this algorithm has shown results comparable or better than those obtained with AdaBoost or the neural net. The training time used by this algorithm is comparable to that used by AdaBoost with decision trees; the algorithm is therefore faster than the neural net in high dimensions. The method has been coded in $\mathrm{C}++$ and included in the StatPatternRecognition package available for free distribution to HEP analysts.

\section{BAGGING DECISION TREES}

The implementation of decision trees used for the proposed algorithm is described in detail in Ref. [8]. The key feature of this implementation is its ability to optimize HEP-specific figures of merit such as the signal significance.

A decision tree, even if it directly optimizes the desired figure of merit, is rarely powerful enough to achieve a good separation between signal and background. The tree produces a set of signal-dominated rectangular regions. Rectangular regions, however, often fail to capture a non-linear structure of data. The mediocre predictive power of a single decision tree can be greatly enhanced by one of the two popular methods for combining classifiers boosting and bagging.

Both these methods work by training many classifiers, e.g., decision trees, on variants of the original training data set. A boosting algorithm enhances weights of misclassified events and reduces weights of correctly classified events and trains a new classifier on the reweighted sample. The output of the new classifier is then used to re-evaluate fractions of correctly classified and misclassified events and update the event weights accordingly. After training is completed, events are classified by a weighted vote of the trained classifiers. AdaBoost, a popular version of this approach, has been shown to produce a high-quality robust training mechanism. Application of AdaBoost to HEP data has been explored in Refs. 7, [8].

In contrast, bagging algorithms [9] do not reweight events. Instead, they train new classifiers on bootstrap replicas of the training set. Each bootstrap replica 10] is obtained by sampling with replacement from the original training set, with the size of each replica equal to that of the original set. After training is completed, events are classified by the majority vote of the trained classifiers. For successful application of the bagging algorithm, the underlying classifier must be sensitive to small changes in the training data. Otherwise all trained classifiers will be similar, and the performance of the single classifier will not be improved. This condition is satisfied by a decision tree with fine terminal nodes. Because of the small node size each decision tree is significantly overtrained; if the tree were used just by itself, its predictive power on a test data set would be quite poor. However, because the final decision is made by the majority vote of all the trees, the algorithm delivers a high predictive power.

Various kinds of boosting and bagging algorithms have been compared in the statistics literature. Neither of these two approaches has a clear advantage over the other. On average, boosting seems to provide a better predictive power. Bagging tends to perform better in the presence of outliers and significant noise [11].

For optimization of the signal significance, however, bagging is the choice favored by intuition. Reweighting events has an unclear impact on the effectiveness of the optimization routine with respect to the chosen figure of merit. While it may be possible to design 
a reweighting algorithm efficient for optimization of a specific figure of merit, at present such reweighting algorithms are not known. Bagging, on the other hand, offers an obvious solution. If the base classifier directly optimizes the chosen figure of merit, bagging is equivalent to optimization of this figure of merit integrated over bootstrap replicas. In effect, the bagging algorithm finds a region in the space of physical variables that optimizes the expected value of the chosen figure of merit - exactly what the analyst is looking for.

Bagging decision trees is certainly not a new item in the statistics research. The only novelty introduced in this note is the decision tree designed for direct optimization of an arbitrary figure of merit, e.g., the signal significance.
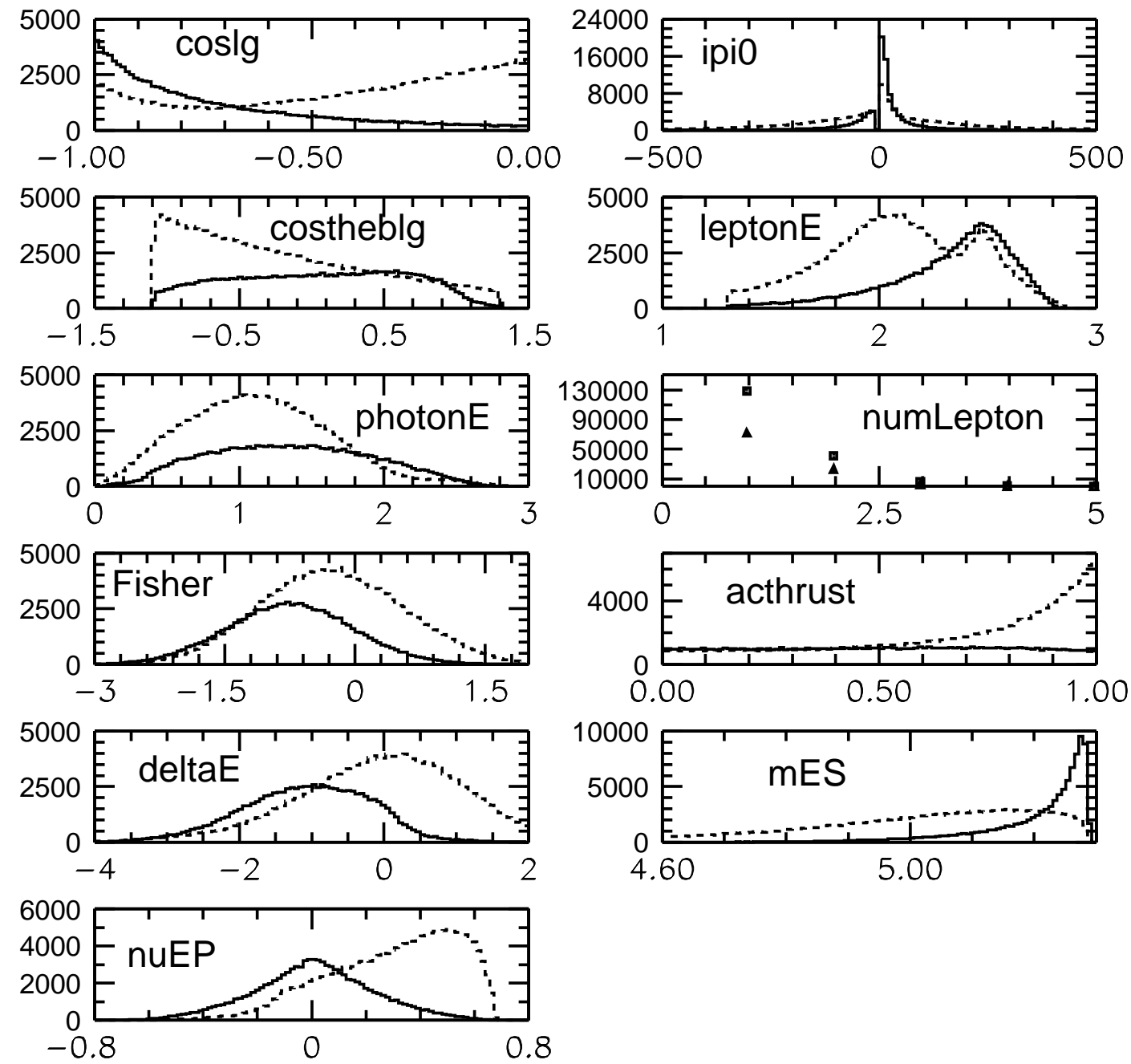

FIG. 1: Separation variables for the $B \rightarrow \gamma l \nu$ analysis. Signal MC is shown with a solid line (triangles in the numLepton plot), and the overall combined background is shown with a dashed line (squares in the numLepton plot). 
TABLE I: Signal significance, $\mathcal{S}_{\text {train }}, \mathcal{S}_{\text {valid, }}$, and $\mathcal{S}_{\text {test }}$, for the $B \rightarrow \gamma l \nu$ training, validation, and test samples obtained with various classification methods. The signal significance computed for the test sample should be used to judge the predictive power of the included classifiers. A branching fraction of $3 \times 10^{-6}$ was assumed for both $B \rightarrow \gamma \mu \nu$ and $B \rightarrow \gamma e \nu$ decays. $W_{1}$ and $W_{0}$ represent the signal and background, respectively, expected in the signal region after the classification criteria have been applied; these two numbers have been estimated using the test samples. All numbers have been normalized to the integrated luminosity of $210 \mathrm{fb}^{-1}$. The best value of the expected signal significance is shown in boldface.

\begin{tabular}{|c|c|c|c|c|c|c|c|c|c|c|}
\hline \multirow{2}{*}{ Method } & \multicolumn{4}{|c|}{$B \rightarrow \gamma e \nu$} & \multicolumn{4}{c|}{$B \rightarrow \gamma \mu \nu$} \\
\cline { 2 - 11 } & $\mathcal{S}_{\text {train }}$ & $\mathcal{S}_{\text {valid }}$ & $\mathcal{S}_{\text {test }}$ & $W_{1}$ & $W_{0}$ & $\mathcal{S}_{\text {train }}$ & $\mathcal{S}_{\text {valid }}$ & $\mathcal{S}_{\text {test }}$ & $W_{1}$ & $W_{0}$ \\
\hline Original method & 2.66 & - & 2.42 & 37.5 & 202.2 & 1.75 & - & 1.62 & 25.8 & 227.4 \\
\hline Decision tree & 3.28 & 2.72 & 2.16 & 20.3 & 68.1 & 1.74 & 1.63 & 1.54 & 29.0 & 325.9 \\
\hline Bump hunter with one bump & 2.72 & 2.54 & 2.31 & 47.5 & 376.6 & 1.76 & 1.54 & 1.54 & 31.7 & 393.8 \\
\hline AdaBoost with binary splits & 2.53 & 2.65 & 2.25 & 76.4 & 1077.3 & 1.66 & 1.71 & 1.44 & 45.2 & 935.6 \\
\hline AdaBoost with decision trees & 13.63 & 2.99 & 2.62 & 58.0 & 432.8 & 11.87 & 1.97 & 1.75 & 41.6 & 523.0 \\
\hline Combiner of background subclassifiers & 3.03 & 2.88 & 2.49 & 83.2 & 1037.2 & 1.84 & 1.90 & 1.66 & 55.2 & 1057.1 \\
\hline Bagging decision trees & 9.20 & 3.25 & $\mathbf{2 . 9 9}$ & 69.1 & 465.8 & 8.09 & 2.07 & $\mathbf{1 . 9 8}$ & 49.4 & 571.1 \\
\hline
\end{tabular}
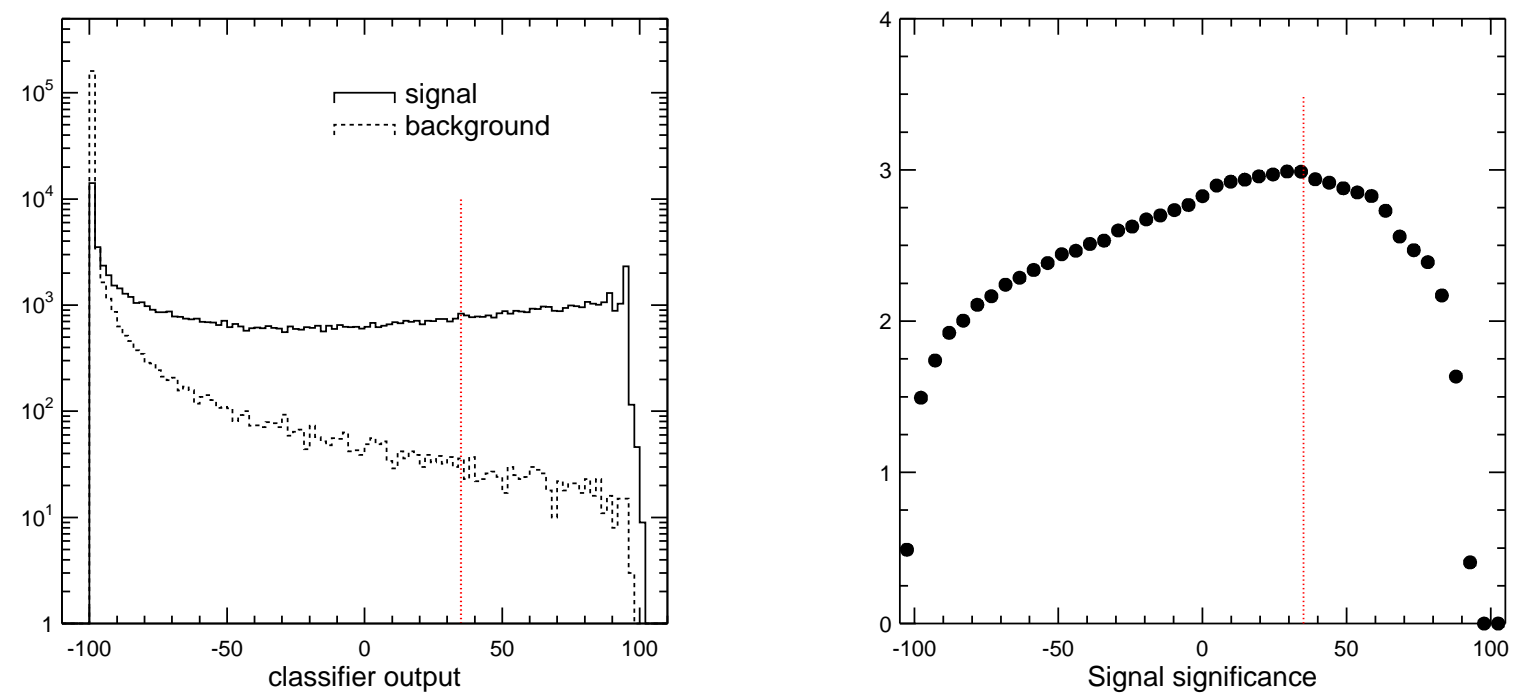

FIG. 2: Output of the bagging algorithm with 100 trained decision trees (left) and the signal significance versus the lower cut on the output (right) for the $B \rightarrow \gamma e \nu$ test sample. The cut maximizing the signal significance, obtained using the validation sample, is shown with a vertical line. 
Performance of the described bagging algorithm has been studied using a search for the radiative leptonic decay $B \rightarrow \gamma l \nu$ at $B_{A} B A R$. Eleven variables used for classification in this analysis are shown in Fig. 1. Several methods have been used to separate signal from background by maximizing the signal significance: an original method developed by the analysts, the decision tree optimizing the signal significance, the bump hunting algorithm, AdaBoost with binary splits, AdaBoost with decision trees optimizing the Gini index, and an AdaBoost-based combiner of background subclassifiers. I also attempted to use a feedforward backpropagation neural network with one hidden layer, but the network was unstable and it failed to converge to an optimum. A more detailed description of this analysis and used classifiers can be found in Ref. [8].

To test the bagging algorithm described in this note, I trained 100 decision trees on bootstrap replicas of the training data. For classification of new data, the trained trees were combined using an algebraic sum of their outputs: if an event was accepted by a tree, the output for this event was incremented by 1 and decremented by 1 otherwise. The minimal size of the terminal node in each tree, 100 events for both $B \rightarrow \gamma e \nu$ and $B \rightarrow \gamma \mu \nu$ channels, was chosen by comparing values of the signal significance computed for the validation data. The size of the trained decision trees varied from 390 to 470 terminal signal nodes in the

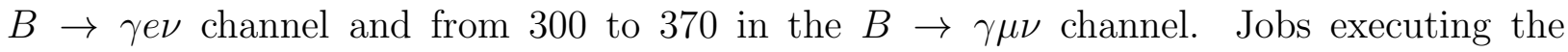
algorithm took several hours in a batch queue at SLAC. To assess the true performance of the method, the signal significance was then evaluated for the test data.

All attempted classifiers are compared in Table I. The output of the described bagging algorithm for the $B \rightarrow \gamma e \nu$ test data is shown in Fig. 2. The bagging algorithm provides the best value of the signal significance. It gives a $24 \%$ improvement over the original method developed by the analysts and shown in the first line of Table I, and a 14\% improvement over AdaBoost with decision trees shown in line 5 of Table I; both numbers are quoted for the $B \rightarrow$ rev channel.

I also used AdaBoost with decision trees optimizing the signal significance and the bagging algorithm with decision trees optimizing the Gini index. The first method performed quite poorly; the signal significance obtained with this method was much worse than that obtained by AdaBoost with decision trees optimizing the Gini index. The bagging algorithm with decision trees optimizing the Gini index showed an $8 \%$ improvement in the $B \rightarrow \gamma e \nu$ signal significance compared to AdaBoost with decision trees optimizing the Gini index. But the signal significance obtained with this method was $9 \%$ worse than that obtained by the bagging algorithm with decision trees optimizing the signal significance. The $14 \% \mathrm{im}-$ provement of the proposed bagging algorithm over AdaBoost with decision trees originated therefore from two sources:

- Using bagging instead of boosting.

- Using the signal significance instead of the Gini index as the figure of merit for the decision tree optimization.

In an attempt to improve the signal significance even further, I used the random forest approach [12], a more generic resampling method. In addition to generating a new bootstrap replica for each tree, I resampled the data variables used to split each node of the tree. Because a bootstrap replica contains on average $63 \%$ of distinct entries from the original set, only 6.9 variables out of 11 were used on average to split the tree nodes. This approach

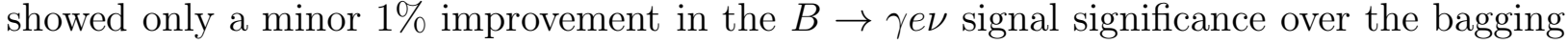
algorithm without variable resampling. 
As shown in Fig. 2, the described bagging algorithm does not provide a good separation between signal and background in terms of the quadratic or exponential classification error. It misclassifies a large fraction of signal events. However, the method does the job it was expected to do - it finds a region in the space of physical variables that, on average, maximizes the signal significance.

\section{SUMMARY}

A bagging algorithm suitable for optimization of an arbitrary figure of merit has been described. This algorithm has been shown to give a significant improvement of the signal significance in the search for the radiative leptonic decay $B \rightarrow \gamma l \nu$ at $B A B A R$. Included in the StatPatternRecognition package [8], this method is available to HEP analysts.

\section{Acknowledgments}

Thanks to Frank Porter for comments on a draft of this note.

[1] R.A. Fisher, The use of multiple measurements in taxonomic problems, Annals of Eugenics 7, 179-188 (1936).

[2] W.S. McCulloch and W. Pitts, A logical calculus of the ideas immanent in nervous activity, Bulletin of Mathematical Biophysics 5, 115-133 (1943); F. Rosenblatt, The Perceptron: A probabilistic model for information storage and organization in the brain, Psychological Review 65, 386-408 (1958); J.J. Hopfield, Neural networks and physical systems with emergent collective computational abilities, Proceedings of the National Academy of Sciences, USA, 79, 2554-2558 (1982); D.E. Rumelhart et al., Learning internal representation by error propagation, Parallel Distributed Processing: Explorations in the Microstructure of Cognition 1, 318-362 (1986); J.A. Anderson and E. Rosenfeld, Neurocomputing. Foundations of Research, MIT Press, 1988.

[3] L. Breiman et al., Classification and Regression Trees, Waldsworth International, 1984.

[4] J. Friedman and N. Fisher, Bump hunting in high dimensional data, Statistics and Computing 9, 123-143 (1999).

[5] Y. Freund and R.E. Schapire, A decision-theoretic generalization of on-line learning and an application to boosting, J. of Computer and System Sciences 55, 119-139 (1997); L. Breiman, Arcing classifiers, The Annals of Statistics 26, 801-849 (1998); R.E. Schapire et al., Boosting the margin: A new explanation for the effectiveness of voting methods, The Annals of Statistics 26, 1651-1686 (1998).

[6] D. Bowser-Chao and D.L. Dzialo, A Comparison of Binary Decision Trees and Neural Networks in Top Quark Detection, Phys. Rev. D47, 1900-1905 (1993); M. Mjahed, Multivariate Decision Tree Designing for the Classification of Multi-Jet Topologies in $e^{+} e^{-}$Collisions, Nucl. Instrum. and Meth. A481, 601-614 (2002); R. Quiller, Decision Tree Technique for Particle Identification, SLAC-TN-03-019, 2003.

[7] B.P. Roe et al., Boosted Decision Trees, an Alternative to Artificial Neural Networks, physics/0408124, 2004. 
[8] I. Narsky, StatPatternRecognition: A C++ Package for Statistical Analysis of High Energy Physics Data, physics/0507143, 2005.

[9] L. Breiman, Bagging Predictors, Machine Learning 26, 123-140 (1996); L. Lam and C.Y. Suen, Application of majority vote to pattern recognition: An analysis of its behavior and performance, IEEE Transactions on Systems, Man, and Cybernetics 27, 553-568 (1997).

[10] B. Efron and R.J. Tibshirani, An Introduction to the Bootstrap, Chapman \& Hall/CRC, 1993.

[11] E. Bauer and R. Kohavi, An empirical comparison of voting classification algorithms: Bagging, boosting, and variants, Machine Learning 36, 105-142 (1999).

[12] L. Breiman, Random Forests, Machine Learning 45, 5-32 (2001). 